\title{
Compare the Clinical Profile of Pediatric Patients in either Deep or Awake State under Sevoflurane Anaesthesia after the Removal of Proseal LMA
}

\author{
Maninder Patni ${ }^{1}$, Uday Pratap ${ }^{2}$, Ruchi Sarawgi ${ }^{3}$, Ruchi Gupta ${ }^{4}$ \\ ${ }^{1}$ Assistant Professor, Department of Anaesthesia, Pacific Medical College, Udaipur, ${ }^{2}$ Assistant Professor, Department of Anaesthesia, Geetanjali Medical \\ College, Udaipur, ${ }^{3}$ Consultant, Arth Diagnostic Centre, Udaipur, ${ }^{4}$ Assistant Professor, Department of Obstetrics \& Gynaecology, Geetanjali Medical Collge, \\ Udaipur.
}

\section{Abstract}

Background: Infraumbilical surgeries in children are usually carried out under spontaneous inhalational technique. The advent of the LMA has ensured that such surgeries can be carried out at a lower concentration of the inhalational anaesthetic. In addition, a caudal block can be given intraoperative to decrease the anaesthetic requirement. Subjects and Methods: Two groups of 30 each by the computer. Group A (Awake Group): LMA was removed in the awake state. Group B (Deep Group): LMA was removed in the deep anaesthetized state. Anaesthesia technique: Patients were given the predetermined concentration of sevoflurane for 10 minutes depending on the group they belonged to. In the deep group patients were given sevoflurane at a concentration of 2-2.2\% for 10 minutes prior to the anticipated end of surgery, while patients in the awake group were given sevoflurane at a concentration of $0.3-0.4 \%$ for 10 minutes prior to the anticipated end of surgery. Results: In the post operative period all 30 patients from the groups- deep and awake were observed during the first minute. In the second minute all 30 patients were still under observation in the deep group while in the awake group 15 patients were transferred to the recovery as they fulfilled the criteria for transfer. Hence after the third minute only patients from the deep group were left for observation in the operating room. Conclusion: LMA-ProSeal can be safely removed during awake and deep states of anaesthesia and neither is associated with an increase in the incidence of complications. Transfer time to the recovery room is however significantly less after awake removal

Keywords: LMA-ProSeal, Sevoflurane.

Corresponding Author: Dr Uday Pratap, Assistant Professor, Department of Anaesthesia, Geetanjali Medical College, Udaipur.

Received: July 2019

Accepted: August 2019

\section{Introduction}

Infraumbilical surgeries in children are usually carried out under spontaneous inhalational technique. The advent of the LMA has ensured that such surgeries can be carried out at a lower concentration of the inhalational anaesthetic. In addition, a caudal block can be given intraoperatively to decrease the anaesthetic requirement. ${ }^{[1]}$

ProSeal-LMA was developed by Dr. Archie Brain in the late nineteen nineties. The Pro-Seal LMA is more useful than the classic LMA in situations where higher airway pressures are required and in cases of difficult airway. ProSeal-LMA is similar to the classic LMA in looks, insertion and quality of airway, but better in terms of seal pressure and protection against gastric contents. ${ }^{[2]}$ Its cuff extends over the posterior surface of the bowl to push the mask anteriorly to sustain airway pressures greater than $30 \mathrm{mmHg}$. A second tube parallel to the ventilation tube passes through the bowl of the mask to the tip of the cuff where it opens at the upper esophageal sphincter. This drain tube separates the respiratory tract from the esophagus. It directs the regurgitated gastric fluid away from the airway and provides a conduit for blind passage of a gastric tube for aspiration of gas or liquid contents from the stomach. ${ }^{[3,4]}$

The manufacturers recommend that the LMA should always be removed in an awake state after the return of protective airway reflexes. This may however carry the risk of respiratory complications such as coughing, breath holding and laryngeal spasm. ${ }^{[5]}$

Removal of the LMA-ProSeal may also be performed when the patient is breathing spontaneously and airway reflexes are depressed i.e. the patient is in a deeply anaesthetized state. This technique provides for smoother emergence and reduces cough and other airway complications such as bronchospasm and laryngospasm. However, this maybe associated with certain disadvantages i.e. the airways remain unprotected from secretions, which if present maybe aspirated. $^{[6]}$

Sevoflurane is the induction agent of choice in children because of fast induction and early recovery. It is less pungent than isoflurane and desflurane and is equivalent to or even superior to halothane for induction. Sevoflurane and halothane are approximately equivalent in terms of airway 
complications during induction though the rate of induction is more rapid with sevoflurane. ${ }^{[7]}$ Data pooled from various studies indicate that there is no difference in the incidence of laryngospasm and bronchospasm, but the incidence of coughing is lower with sevoflurane. Sevoflurane and halothane both produce a dose related respiratory depression, however halothane produces a decrease in the tidal volume and an increase in the respiratory rate. An important concern with sevoflurane is the apparently higher incidence of agitation as compared with halothane. ${ }^{[8,9]}$

Most of the studies have analysed removal of the LMAClassic in the anaesthetized and awake states using various inhalational agents. Because of the various advantages of the LMA-ProSeal over the LMA-Classic and the rapid recovery profile of sevoflurane we decided to take up this study to evaluate and compare the clinical parameters and various complications associated with the removal of LMA-ProSeal under awake and anaesthetized states in children aged between the ages of 4-12 years. Hence the aim and objective of the study was to comparison of the clinical profile of paediatric patients after ProSeal removal in deep and awake state under sevoflurane anaesthesia.

\section{Subjects and Methods}

The study was conducted in the Department of Anaesthesia and Critical Care, after approval from the ethics committee and written informed consent from the patients.

Patients selected for the study met the following criteria:

\section{Inclusion Criteria}

1. Children 4-12 years of age

2. ASA-I

3. Patients with minimum 2 hours of fasting after clear fluids and 6 hours fasting after light meals

4. Elective surgery

5. Duration of surgery less than 2 hours

6. Infraumblical surgery

\section{Exclusion Criteria}

1. Age $<4$ years or $>12$ years

2. History of upper respiratory tract infection (URI) in the last 3 weeks

3. History of Asthma

4. Restricted mouth opening

5. Duration of surgery $>2$ hours

A thorough pre-anaesthetic evaluation was done for all the patients. Weight and height of the patients was noted and BMI was calculated. After considering the exclusion criteria, patients were accepted and their parent/guardian was explained the procedure. An informed written consent was taken from the parent/guardian. Patients were allocated to one of the two groups on a random basis. Sixty random numbers were generated and allotted to two groups of 30 each by the computer. These random numbers were kept in a sealed envelope.

GROUP A (AWAKE GROUP): LMA was removed in the awake state.

GROUP B (DEEP GROUP): LMA was removed in the deep anaesthetized state.

The patients were kept fasting for 6 hours prior to surgery. Clear fluids were allowed uptil 2 hours preoperatively. EMLA cream was applied on the chosen site atleast 1 hour prior to the surgery. After placing the monitors, heart rate, non invasive blood pressure (NIBP), oxygen saturation and ECG were continuously monitored and recorded at regular intervals. Initial values of heart rate, NIBP, oxygen saturation on room air and ECG were noted and intravenous access was secured with a $22 \mathrm{G}$ cannula. Premedication was done with intravenous fentanyl at a dose of $1 \mu \mathrm{g} / \mathrm{kg}$.

Sevoflurane $8 \%$ in $50 \%$ nitrous oxide was used for induction. This concentration of sevoflurane was delivered till jaw relaxation occurred. After achievement of jaw relaxation the concentration of sevoflurane delivered was reduced to $2-3 \%$. This was followed by insertion of a suitable size of the ProSeal LMA.

The patient was maintained on spontaneous ventilation and assisted ventilation was given whenever the $\mathrm{ETCO}_{2}$ exceeded $40 \mathrm{mmHg}$. The concentration of sevoflurane delivered was adjusted according to the clinical signs. $\mathrm{F}_{\mathrm{I}} \mathrm{O}_{2}$ was kept at $50 \%$ throughout the surgery. Analgesia was provided with a caudal block using $0.5-1 \mathrm{ml} / \mathrm{kg}$ of $0.25 \%$ bupivacaine, depending on the site of the surgery.

Patients were given the predetermined concentration of sevoflurane for 10 minutes depending on the group they belonged to. In the deep group patients were given sevoflurane at a concentration of $2-2.2 \%$ for 10 minutes prior to the anticipated end of surgery, while patients in the awake group were given sevoflurane at a concentration of $0.3-0.4 \%$ for 10 minutes prior to the anticipated end of surgery. After this the ProSeal LMA was removed after thorough suctioning. In case of removal in the deep anaesthetized state the jaw was lifted and $100 \%$ oxygen was given via face mask till the patient regained jaw tone. The patients were transferred to the recovery after either they vocalized or regained their jaw tone.

\section{Intraoperatively monitored parameters:}

a. Pulse

b. Respiratory Rate

c. Non invasive blood pressure

d. $\mathrm{O}_{2}$ saturation $\left(\mathrm{SpO}_{2}\right)$

e. Inspired fraction (Fi) and expired fraction $(\mathrm{Fe})$ of sevoflurane

f. Minimum Alveolar Concentration (MAC) of sevoflurane These parameters were monitored at intervals of 5 minutes each till the end of surgery.

\section{Postoperatively monitored parameters:}

a. Pulse

b. Respiratory Rate

c. Non invasive blood pressure

d. $\mathrm{SpO}_{2}$

e. $\mathrm{Fi}$ and $\mathrm{Fe}$ of sevoflurane

f. Minimum Alveolar Concentration ( MAC) of sevoflurane These parameters were monitored every minute till the patient became eligible to be transferred to the recovery room. The time taken from the removal of the Pro Seal LMA till transfer of patients to the recovery room was also noted. 


\section{Statistical Analysis}

Frequency distributions and their percentage were applied for categorical variables like sex, and complications. To observe the difference between the groups as regards the continuous variables (age, BMI, pulse, BP, respiratory rate) student t-test (independent) were applied. Chi-square test was applied to see the association between two groups in cases of categorical variables. p- value $<0.05$ was considered as statistically significant. SPSS 10.0 statistical software was used for analysis.

\section{Results}

Sixty patients of either sex in the age group of 4-12 years belonging to ASA -I, scheduled for infra-umblical surgeries were included in this prospective randomized study. This study was carried out to compare the clinical profile of paediatric patients in either deep or awake state under sevoflurane anaesthesia after the removal of ProSeal LMA. The patients were divided into 2 groups of 30 each. In Group A, LMA was removed in the awake state ( $\mathrm{Fi}$ sevoflurane at $0.3-0.4 \%$ ) and in Group B, LMA was removal in a deep anaesthetized state (Fi sevoflurane at 2-2.2\%).

The mean age among the two groups was comparable $(\mathrm{p}=0$ .599). There were six children in the deep group and nine children in the awake group in the four year age group. There were seventeen children in the deep group twelve children in the awake group in the five year age group. There were six children in the deep group seven children in the awake group in the six year age group. There was one child in the deep group and two children in the awake group in the seven year age group. The mean age (in years) in the deep group as well as the awake group was 5.067. Thus there is no significant difference between the two groups in the mean age distribution.

Table 1: Post-operative Mean Fe of Sevoflurane

\begin{tabular}{|c|c|c|c|c|c|c|c|c|}
\hline \multicolumn{2}{|l|}{ Group } & $1 \mathrm{~min}$ & $2 \mathrm{~min}$ & $3 \mathrm{~min}$ & $4 \mathrm{~min}$ & $5 \mathrm{~min}$ & $6 \mathrm{~min}$ & $7 \mathrm{~min}$ \\
\hline \multirow{3}{*}{ Deep } & $\mathrm{N}$ & 30 & 30 & 30 & 29 & 25 & 12 & 4 \\
\hline & Mean & 1.5063 & 0.6297 & 0.4233 & 0.281 & 0.134 & 0.106 & 0.195 \\
\hline & SD & 0.3015 & 0.384 & 0.1461 & 0.153 & 0.141 & 0.132 & 0.023 \\
\hline \multirow[t]{3}{*}{ Awake } & $\mathrm{N}$ & 30 & 15 & 2 & & & & \\
\hline & Mean & 0.1953 & 0.1407 & 0.19 & & & & \\
\hline & SD & 0.13706 & 0.11405 & 0.04243 & & & & \\
\hline p Value & & 0.000 & 0.000 & 0.034 & & & & \\
\hline
\end{tabular}

$\mathrm{N}=$ Number of patients under observation in the operating room before transfer to the recovery room

In the postoperative period all 30 patients from the groups deep and awake, were observed during the first minute. In the second minute all 30 patients were still under observation in the deep group while in the awake group 15 patients were transferred to the recovery room as they fulfilled the criteria for transfer. Hence during the second minute only 15 patients in the awake group were under observation. During the third minute all 30 patients from the deep group were under observation, while 13 more patients from the awake group were transferred to the recovery room. After the third minute, all thirty patients in the deep group were still under observation, while the remaining two patients in the awake group were also transferred. Hence after the third minute only patients from the deep group were left for observation in the operating room.

The mean pulse rate during the first minute postoperatively was 92.43 per min in the deep group and 105.3 per min in the awake group. This difference was statistically significant $(p=0.00)$. The mean pulse rate during the second minute post-operative was 90.4 per min in the deep group and 102.67 per min in the awake group. This difference was statistically significant $(\mathrm{p}=0.00)$. The mean pulse rate during the third minute post-operative was 90 in the deep group and 96 in the awake group. This difference was not statistically significant $(\mathrm{p}=0.09)$.

The mean post-operative systolic BP during the first minute was $92.03 \mathrm{~mm} \mathrm{Hg}$ in the deep group and $95.47 \mathrm{~mm} \mathrm{Hg}$ in the awake group. This value was statistically significant $(p=0.011)$. The mean post-operative systolic BP during the second minute was $92.37 \mathrm{~mm} \mathrm{Hg}$ in the deep group and 96.2 $\mathrm{mm} \mathrm{Hg}$ in the awake group. This value was statistically significant $(\mathrm{p}=0.000)$. The mean post-operative systolic BP during the third minute was $91.73 \mathrm{~mm} \mathrm{Hg}$ in the deep group and $96 \mathrm{~mm} \mathrm{Hg}$ in the awake group. This value was not statistically significant $(\mathrm{p}=0.083$ )

The mean post-operative diastolic BP during the first minute was $61.37 \mathrm{mmHg}$ in the deep group and $61.13 \mathrm{mmHg}$ in the awake group. This value was not statistically significant $(\mathrm{p}=.896)$. The mean post-operative diastolic BP during the second minute was $59.87 \mathrm{mmHg}$ in the deep group and $63.6 \mathrm{mmHg}$ in the awake group. This value was statistically significant $(\mathrm{p}=0.000)$. The mean post-operative diastolic BP during the third minute was $60.13 \mathrm{mmHg}$ in the deep group and $65 \mathrm{mmHg}$ in the awake group. This value was statistically significant $(\mathrm{p}=.021)$

The mean respiratory rate of patients during the first minute post-operative was 21.87 per $\min$ in the deep group and 23.23 per min in the awake group. This difference was statistically significant $(\mathrm{p}=0.002)$. The mean respiratory rate during the second minute post-operative was 21.83 per min in the deep group and 23.07 per min in the awake group. This difference was statistically significant $(\mathrm{p}=0.003)$. The mean respiratory rate during the third minute post-operative was 21.57 per min in the deep group and 23.5 per min in the awake group. This difference was statistically significant $(\mathrm{p}=0.008)$.

The mean $\mathrm{Fe}$ of sevoflurane during the first minute postoperative was 1.5063 in the deep group and 0.1953 in the awake group. This difference was statistically significant(0.000). The mean Fe of sevoflurane during the second minute post-operative was 0.6297 in the deep group and 0.1407 in the awake group. This difference was 
statistically significant (0.000). The mean Fe of sevoflurane during the third minute post-operative was 0.4233 in the deep group and 0.19 in the awake group. This difference was statistically significant (0.034).

The mean Fi of sevoflurane during the first minute postoperative was 1.9 in the deep group and 0.03 in the awake group. This difference was statistically significant (0.000).
The mean Fi of sevoflurane during the second minute postoperative was 0.59 in the deep group and 0.002 in the awake group. This difference was statistically significant $(0.001)$. The mean Fi of sevoflurane during the third minute postoperative was 0.04 in the deep group and 0 in the awake group. This difference was not statistically insignificant (0.410).

Table 2: Post-operative Mean Fi of Sevoflurane

\begin{tabular}{|c|c|c|c|c|c|c|c|c|}
\hline \multicolumn{2}{|c|}{ GROUP } & \multirow{2}{*}{$\begin{array}{l}1 \text { min } \\
30\end{array}$} & \multirow{2}{*}{$\frac{2 \text { min }}{30}$} & \multirow{2}{*}{$\begin{array}{l}3 \text { min } \\
30\end{array}$} & \multirow{2}{*}{$\begin{array}{l}4 \mathrm{~min} \\
29\end{array}$} & \multirow{2}{*}{$\begin{array}{l}5 \text { min } \\
15\end{array}$} & \multirow{2}{*}{$\begin{array}{l}6 \text { min } \\
12\end{array}$} & \multirow{2}{*}{$\begin{array}{l}7 \mathrm{~min} \\
4\end{array}$} \\
\hline & $\mathrm{N}$ & & & & & & & \\
\hline \multirow[t]{3}{*}{ Deep } & Mean & 1.9 & 0.59 & 0.04 & 0.04 & 0.02 & 0 & 0 \\
\hline & SD & 0.23 & 0.6583 & 0.065 & 0.062 & 0.065 & 0.003 & 0 \\
\hline & $\mathrm{N}$ & 30 & 15 & 2 & & & & \\
\hline \multirow[t]{2}{*}{ Awake } & Mean & 0.03 & 0.002 & 0 & & & & \\
\hline & SD & 0.059 & 0.0041 & 0 & & & & \\
\hline P Value & & 0.000 & 0.001 & 0.410 & & & & \\
\hline
\end{tabular}

$\mathrm{N}=$ Number of patients under observation in the operating room before transfer to the recovery room

In children, as the characteristics are different from those of adults and some children do not respond well to verbal commands, it is difficult to judge whether the child is awake or lightly anaesthetised. Moreover, although the LMA is well tolerated in adults, this is not always the case in children. In some children, such as those with asthma, removal may be safer during deeper anaesthesia as this reduces the incidence of coughing, laryngospasm, biting and hypoxia. ${ }^{[5]}$

The main concerns of removal of an airway device in a deeper plane of anaesthesia include prolonged airway obstruction and delayed return of airway reflexes. An adequate concentration of sevoflurane with its rapid recovery profile is useful in this regard. Rapid recovery with sevoflurane was seen by Robert D Valley et $\mathrm{al}^{12}$ who noted that after deep tracheal extubation of children using sevoflurane or isoflurane, the return to arousable state occurred more quickly with sevoflurane. Fink et al reported a $60 \%$ reduction in early recovery time (the time to follow commands) in patients awakening from sevoflurane versus isoflurane anaesthesia.

In our study, the concentration of sevoflurane used for removal of the LMA ProSeal in the deep state was 2-2.2\%. All the patients in the deep group were able to preserve their airway patency with slight jaw lift and $100 \%$ oxygen. The mean time taken for maintaining airway patency without any support after removal of the LMA ProSeal was 5.37 minutes. This was also taken as the transfer time of patients from the operating room to the recovery room. These observations are similar to those of Jeong Rim Lee et $\mathrm{al}^{37}$, who in their study on minimum alveolar concentration (MAC) of sevoflurane concluded that LMA removal may be accomplished without coughing, moving or any other airway complications at a sevoflurane concentration of $2.17 \%$ in $95 \%$ of children. Similar to our study, they also noted that patients were able to maintain patency of their airway without support within 5 minutes of removal of the LMA. Yon Hee Shim et al ${ }^{12,13}$ concluded that the end tidal concentration of sevoflurane required to achieve successful LMA removal in 50\% adults was $0.99 \%$ and in $95 \%$ adults was $1.18 \%$. Thus in comparison to our study, their results suggest a lesser requirement of sevoflurane for deep removal of the LMA. But unlike us, they studied the adult age group. Adults tend to tolerate the LMA without any difficulty even in a lighter plane of anaesthesia, unlike children.

Proponents of awake removal of the LMA claim that fast return of airway reflexes is critical in surgeries that irritate the upper airway or increase secretions. This argument in favour of awake removal of the LMA has been supported by Dolling $\mathrm{S}$ et $\mathrm{al}^{14}$ who compared deep versus awake removal of the LMA in paediatric dental day care surgery and observed that peripheral oxygen saturation was significantly lower in the deep group as compared to the awake group.

In our study we used a standard anaesthetic regimen so that the groups were as comparable as possible. Intravenous induction agents such as thiopentone and propofol are known to have differing effects on airway excitability and this might have interfered with our study. We therefore decided to use sevoflurane and nitrous oxide for induction in all patients. After the child was anaesthetised we used fentanyl in a dose of $1 \mu \mathrm{g} / \mathrm{kg}$ to suppress airway reflexes before insertion of the LMA ProSeal. A caudal block was given to provide analgesia for the operative site and hence decrease the overall anaesthetic requirement.

In both the groups all the patients were males, the reason being that surgeries chosen for the study were infraumbilical e.g. uethroplasty, inguinal hernia repair and orchidopexy.

The mean BMI in the awake group was $21.20 \mathrm{~kg} / \mathrm{m}^{2}$ while the BMI in the deep group was $21.10 \mathrm{~kg} / \mathrm{m}^{2}$. Both the groups were comparable with respect to the BMI.

In the post operative period all 30 patients from both the groups were monitored in the operating room. None of the patients was transferred to the recovery room in the first minute. In the second minute all 30 patients were still under observation in the deep group, while in the awake group 15 patients were transferred to the recovery as they had fulfilled the criteria for transfer. During the third minute all 30 patients from the deep group were still under observation, while 13 more patients from the awake group were transferred to the recovery room. After the third minute, all patients from the deep group were still under observation, while from the awake group all patients had been transferred to the recovery room. Therefore the initial 3 minutes after removal of the LMA have been considered as critical for comparison of the two groups. 
In our study we have monitored and compared the postoperative pulse, blood pressure and respiratory rate in the two groups, from the time of removal of LMA till transfer of patients to the recovery room. We wanted to see if there was any significant difference in the haemodynamic profile between the two groups. We could not find any other study which has compared haemodynamic parameters in patients during awake and deep removal of LMA.

The mean pulse rate in the first and second minutes postoperatively was 92.43 per $\min$ and 90.4 per min respectively in the deep group and 105.3 per min and 102.67 per min respectively in the awake group. The difference between the groups was statistically significant. By the third minute the difference between the groups had decreased and was not significant. The difference in pulse rate in the first 2 minutes may be attributable to a lighter plane as well as anxiety associated with removal of the LMA-ProSEAL in the awake state. None of the studies available have compared postoperative pulse rate between the deep and the awake groups.

The mean systolic BP in the first and second minutes postoperatively was $92.03 \mathrm{mmHg}$ and $92.37 \mathrm{mmHg}$ respectively in the deep group and $95.47 \mathrm{mmHg}$ and 96.2 $\mathrm{mmHg}$ respectively in the awake group. The difference between the groups was found to be significant. By the third minute the difference between groups was no longer statistically significant. The mean diastolic BP in the second and third minutes was significantly greater in the awake group as compared to the deep group. The difference in BP maybe attributed to a decrease in systemic vascular resistance and myocardial contractility caused by a higher concentration of sevoflurane used in the deep group.

The mean respiratory rate in the awake group was significantly greater than the deep group during the 3 minutes in which both groups of patients were observed in the operating room. This difference may be attributed to the respiratory depressant effect of sevoflurane.

The postoperative mean Fe of sevoflurane at the first, second and third minutes was 1.5063, 0.6297 and 0.4233 respectively in the deep group and $0.1953,0.1407,0.19$ respectively in the awake group. The difference in $\mathrm{Fe}$ was statistically significant at all the three minutes. This can be attributed to the fact that patients in the deep group were given a higher inspired concentration of sevoflurane as compared to the awake group.

The postoperative mean Fi of sevoflurane at the first, second and third minutes in the deep group was 1.9, 0.59 and 0.04 respectively and in the awake group was $0.03,0.002$ and 0.00 respectively. The difference in Fi between the groups was statistically significant at the first and second minutes. By the third minute Fi of the deep group had decreased and there was no significant difference between the groups.

The mean duration of surgery in the awake group was 59.00 minutes while in the awake group was 48.83 minutes. The difference between the groups was not found to be statistically significant ( $\mathrm{p}=0.063$ ).

The mean transfer time from the operating room to the recovery room was 5.37 minutes in the deep group and 1.57 minutes in the awake group. This difference in transfer time between the two groups was significant $(p=0.000)$. There are not many studies which have compared the difference in transfer times of patients after deep and awake removal of LMA. In a study carried out by Robert D Valley et $\mathrm{al}^{41}$ the mean time taken by patients for maintenance of airway without support after removal of the LMA in a deep state was 5 minutes, which is similar to our study. In our study we considered the patients for transfer after they responded to verbal commands or were able to maintain their jaw tone. Therefore an important finding of our study was that patients who had their LMAs removed in the awake state could be transferred almost 4 minutes earlier than those in whom the LMA was removed while they were deep.

The hemodynamic parameters such as pulse, blood pressure and respiratory rate were significantly more when the LMAProSeal was removed in the awake state, but this was not found to be of clinical significance.

We therefore recommend that the LMA-ProSeal may be removed in either an awake or deep state of anaesthesia. In operating rooms where time is a constraint and a quicker turnover is the need of the hour the LMA-ProSeal can be safely removed when the patient is awake.

\section{Conclusion}

There were no significant differences between the groups as regards the demographic profile, age distribution, sex distribution and BMI. Hence we conclude that LMA-ProSeal can be safely removed during awake and deep states of anaesthesia and neither is associated with an increase in the incidence of complications. Transfer time to the recovery room is however significantly less after awake removal.

\section{References}

1. Ahuja S, Aggarwal M, Joshi N, Chaudhry S, Madhu S: Efficacy of caudal clonidine and fentanyl on analgesia, neuroendocrine stress response and emergence agitation in children undergoing lower abdominal surgeries under general anaesthesia with sevoflurane. Journal of clinical and diagnostic research: JCDR 2015, 9:UC01.

2. Cook T, Howes B: Supraglottic airway devices: recent ad vances. Continuing Education in Anaesthesia, Critical Care and Pain 2011, 11:56-61.

3. Brain A: Laryngeal mask with large-bore gastric drainage. Google Patents, 2004.

4. Ramaiah R, Das D, Bhananker SM, Joffe AM: Extraglottic airway devices: A review. International journal of critical illness and injury science 2014, 4:77

5. Lee J-R, Kim S-D, Kim C-S, Yoon T-G, Kim H-S: Minimum alveolar concentration of sevoflurane for laryngeal mask airway removal in anesthetized children. Anesthesia \& Analgesia 2007, 104:528-31.

6. Gal JS, Yudkowitz FS, Rothschild MA: Pediatric Otolaryngology. Anesthesiology and Otolaryngology: Springer, 2013. pp. 333-64.

7. Muzi M, Robinson B, Ebert T, O'brien T: Induction of anesthesia and tracheal intubation with sevoflurane in adults. Anesthesiology: The Journal of the American Society of Anesthesiologists 1996, 85:53643.

8. Hikasa Y, Ohe N, Takase K, Ogasawara S: Cardiopulmonary effects of sevoflurane in cats: comparison with isoflurane, halothane, and enflurane. Research in veterinary science 1997, 63:205-10.

9. Hikasa Y, Okuyama K, Kakuta T, Takase K, Ogasawara S: Anesthetic potency and cardiopulmonary effects of sevoflurane in goats: comparison with isoflurane and halothane. Canadian Journal of Veterinary Research 1998, 62:299.

10. Park J-S, Kim K-J, Oh J-T, Choi E-K, Lee J-R: A randomized controlled trial comparing Laryngeal Mask Airway removal during adequate anesthesia and after awakening in children aged 2 to 6 years. Journal of clinical anesthesia 2012, 24:537-41.

11. Hall B-C, Hannley M, Durham N: 2007 COMBINED SECTIONS 
MEETING PROGRAM.

12. Valley RD, Ramza JT, Calhoun P, Freid EB, Bailey AG, Kopp VJ,

Georges LS: Tracheal extubation of deeply anesthetized pediatric

patients: a comparison of isoflurane and sevoflurane. Anesthesia \& Analgesia 1999, 88:742-5.

13. Shim YH, Shin CS, Chang CH, Shin Y-S: Optimal end-tidal sevoflurane concentration for the removal of the laryngeal mask airway in anesthetized adults. Anesthesia \& Analgesia 2005, 101:1034-7.

14. Malhotra N: General anaesthesia for dentistry. Indian J Anaesth 2008, 52:725-37.

Copyright: () the author(s), publisher. Academia Anesthesiologica International is an Official Publication of "Society for Health Care \& Research Development". It is an open-access article distributed under the terms of the Creative Commons Attribution Non-Commercial License, which permits unrestricted non-commercial use, distribution, and reproduction in any medium, provided the original work is properly cited.

How to cite this article: Patni M, Pratap U, Sarawgi R, Gupta R. Compare the Clinical Profile of Pediatric Patients in Either Deep or Awake State Under Sevoflurane Anaesthesia After The Removal of Proseal LMA. Acad. Anesthesiol. Int. 2019;4(2):136-141.

DOI: dx.doi.org/10.21276/aan.2019.4.2.32

Source of Support: Nil, Conflict of Interest: None declared. 